\title{
A QUANTITATIVE ANALYSIS OF PREVENTIVE MEASURES TO MITIGATE THE INFECTIOUS DISEASES USING SIR MODEL BY OPTIMAL CONTROL TECHNIQUE
}

\author{
Jakia Sultana, Samiha Islam Tanni and Shamima Islam
}

\begin{abstract}
Optimal Control Problem with the state equations which describes the standard SIR Model is studied here. we considered the SIR Model with vaccination and without vaccination. We formulated an optimal control problem and derived necessary conditions. Existence of the state and the objective functional are also verified. We also characterized the optimal control by Pontryagin's maximum principle which minimizes the number of infected individuals and cost of vaccination over some finite period. Whenever the vaccination is carried out for a long period of time, the simulated result effectively works for diseases with high transmission rate. Observations from the numerical simulation reveals that the infectious diseases are most successfully controlled whenever control strategies were adopted at early stages.
\end{abstract}

Index Terms-SIR Model, Optimal control, Vaccination as control, Existence of the state, Existence of the objective functional, Pontryagin's Maximunm Principle.

\section{INTRODUCTION}

$\mathbf{N}$ Ow-a-days infectious diseases are being studied extensively to improve control over them in order to mitigate and ultimately to eradicate all of the infectious diseases. Appropriate proactive measures, i.e. vaccination, treatment, awareness campaign etc can help in reduction of infectious diseases towards eradication, especially of those which can be combated with both cure and immunization by vaccination. On the other hand, lack of adequate measures and timely interventions can result in epidemic spread of particular infectious diseases.

There are several control measures that might reduce susceptibility and number of infected individuals. The

This paper was received on 31 March 2019 and accepted on 25 June 2019. This work was supported financially by GUBJSE

Jakia Sultana is with the Department of Computer Science and Engineering, Green University of Bangladesh, Dhaka, Bangladesh. E-mail: jakia@cse.green.edu.bd.

Samiha Islam Tanni is with the Department of Computer Science and Engineering, Green University of Bangladesh, Dhaka, Bangladesh. E-mail: samiha@cse.green.edu.bd.

Shamima Islam is with the Department of Computer Science and Engineering, Green University of Bangladesh, Dhaka, Bangladesh. E-mail: shamima@cse.green.edu.bd. effective control strategies are selected in consideration with the nature of the disease, the hosts and the scale of epidemic. In most of the cases, prevention of a disease is cheaper than curing it. While some diseases like malaria, cold etc have no alternatives to treatment, some diseases like polio, must be prevented via vaccination as there is no cure for them. Generally, infectious diseases, i.e. Cholera, Measles, Influenza and Tuberculosis can both be cured by treatment and immunized through vaccination [1]. Mathematical models have become very useful tool for analyzing and controlling these diseases as they can effectively optimize the limited resources when deciding control measures. They can also accurately compare among the implementation of various control programs like treatment and prevention. The most important of all is to select the appropriate program maintaining all the pre-assumed criterions. Granich et. Al. [2] have suggested universal HIV testing based on results from the analysis and simulations of their HIV model. Also an improved Hepatitis B virus (HBV) model was proposed by Wang. et. al. [3] for the treatment of the disease.

Among various mathematical apparatus, optimal control theory is gaining popularity for its effectiveness and simplicity. It is being successfully used whenever selecting appropriate control measures from available treatment and vaccination options. For example, optimal control theory was used by Gaff and Schaffer [1] to select control measures in order to reduce the number of infected population. Zaman et. al. [4] used optimal control theory concentrated on Susceptible Infected Recovered (SIR) model to select vaccination as control measure. Again, it has been applied by Kirschner et. at. [5] for determining treatment strategy for the administration of anti retroviral drug to HIV positive individuals. Fister and Donnelly [2] also effectively used optimal control theory to determine the condition for elimination of the tumor cells in cancer patients.

In this paper, we have not considered any specific disease rather we chose to propose optimal control technique using 
SIR model as a selection tool in general. We used optimal control strategies in the form of vaccination and observed an increase in the number of recovered individuals.

The following section discusses optimal control theory with standard SIR model. Formulation of the specific model with vaccination and without vaccination along with existing optimal control theory is discussed in the II section. Characterization of the optimal control problem is discussed in the IV section. Numerical results with final analysis are provided in section VI. Finally, the paper ends with a brief conclusion.

\section{MOdEL FORMULATION}

There are various models for describing epidemics with different properties in consideration with mortality, immunity, recovery etc [1], [6]. We will consider a standard SIR model with variable total population. To do this, we use a control $u(t)$ in our model. Let $u(t)$ be the percentage of susceptible individuals being vaccinated per unit of time. This percentage is a function of time. Hence, the optimal control (vaccination) strategy is to minimize the infected individuals and to maximize the total number of recovered individuals. Here we formulate our SIR model following [7]

\section{A. Discussion about Classes}

We assume that an individual can be infected only through contacts with infectious individuals and that also that immunity is permanent. We also consider the SIR epidemic model with variable total population size $(N)$. The population is divided into three disease states compartments which are given below:

- Susceptible class $(S)$ : Susceptible class consists of the individuals who are not infected but can be affected by the disease or infection. Individuals are borne into the susceptible class.

- Infectious class $(I)$ : Infectious class consists of the individuals who spread the disease to susceptible and remain in the infectious class for a specified period of time (the infectious period) before moving into the recovered class.

- Recovered class $(R)$ : Recovered class consists of the individuals who were affected by the disease but recovered from the disease or infection.

\section{B. SIR Model without Vaccination:}

In SIR model, the total population is subdivided into three compartments namely, susceptible (S), infectious (I), recovered $(\mathrm{R})$ and the total population can be represented by $N(t)=S(t)+I(t)+R(t)$. the corresponding model in the form of the following system of non-linear differential equation will be considered as the standard SIR Model:

$$
\begin{aligned}
S^{\prime}(t) & =b N(t)-g S(t) I(t)-d s(t) \\
I^{\prime}(t) & =g S(t) I(t)-(\alpha+m+d) I(t) \\
R^{\prime}(t) & =\alpha I(t)-d R(t) \\
N^{\prime}(t) & =(b-d) N(t)-m I(t) .
\end{aligned}
$$

with initial conditions,

$$
S(0)=S_{0} \geq 0, I(0)=I_{0} \geq, R(0)=R_{0} \geq 0, N(0)=N_{0}
$$

Here, The parameter $\mathbf{g}$ is the disease contact rate or transmission rate between the susceptible and infected classes. So the incidence of the disease is described by the term $g S(t) I(t)$. Here $\mathbf{b}$ denotes the natural birth rate and $\mathbf{d}$ indicates the natural death rate. Moreover, $\alpha$ represents the rate at which the infectious individuals become recovered and $\mathbf{m}$ indicates the disease induced death rate.

\section{SIR Model with Vaccination:}

Here the imposing control parameter in the SIR model will be defined as vaccination which may help to control the spread of the infectious disease. Let the control parameter be considered as $u(t)$ which represents the percentage of susceptible individuals keeping under vaccination per unit of time. Then the vaccinated people enters into the recovered class $(R)$. The vaccinated people can be represented by $u(t) S(t)$ over time $t$. Since it is impossible to vaccinate the entire susceptible population, so the control is kept under bound with $0 \leq u(t) \leq 0.9$.

Taking all the above considerations, we rewrite the SIR model with control as follows:

$$
\begin{aligned}
S^{\prime}(t) & =b N(t)-g S(t) I(t)-d s(t)-u(t) S(t) \\
I^{\prime}(t) & =g S(t) I(t)-(\alpha+m+d) I(t) \\
R^{\prime}(t) & =\alpha I(t)-d R(t)+u(t) S(t) \\
N^{\prime}(t) & =(b-d) N(t)-m I(t)
\end{aligned}
$$

with initial conditions,

$S(0)=S_{0} \geq 0, I(0)=I_{0} \geq, R(0)=R_{0} \geq 0, N(0)=N_{0}$

\section{Objective Functional:}

Neilan and Lenhart in [8] proposed an optimal control problem to determine the vaccination strategy over a certain vaccination interval $[0, \mathrm{~T}]$. The aim is to determine the vaccination technique $\mathbf{u}$ to minimize the objective functional.

$$
J(u)=\int_{0}^{T} A I(t)+u^{2}(t) d t
$$

Our aim is to minimize the number of infectious individuals and minimize the total cost of vaccination over $T$ years. The problem is defined as:

$$
\min _{u} \int_{0}^{T} A I(t)+u^{2}(t) d t
$$

and the control set is defined as

$U=\{u:[0, \mathrm{~T}] \rightarrow[0,0.9] \mid u$ is piecewise continuous function $\}$ It is considered that for $u=0$, there are no vaccine and $u=1$ means that all susceptible people are vaccinated. 


\section{Existence of the Optimal Control}

To prove the existence of the optimal control, existence of the state and existence of the objective functional need to be shown.

\section{A. Existence of the State:}

We consider the state equation (2) with initial condition (3). From fourth equation of syestem (2) we can write,

$$
N^{\prime}(t) \leq(b-d) N(t)
$$

so we have

$$
N(t) \leq N_{0} e^{(b-d) T}=V_{1} \in R_{+}
$$

and

$$
\lim _{t \rightarrow \infty} \sup N(t) \leq V_{1} .
$$

which conclude $S(t), I(t), R(t) \leq V_{1}$ as $t \rightarrow \infty$ Then, we can rewrite (1) in the following form:

$$
\phi_{t}=B \phi+F(\phi)
$$

where, $\phi_{t}$ denotes the derivative of $\phi$ with respect to time $t$ and

$$
\begin{gathered}
\phi=\left[\begin{array}{c}
S(t) \\
I(t) \\
R(t) \\
N(t)
\end{array}\right], \phi_{t}=\left[\begin{array}{c}
\frac{d S}{d t} \\
\frac{d I}{d t} \\
\frac{d R}{d t} \\
\frac{d N}{d t}
\end{array}\right] . \\
F(\phi)=\left[\begin{array}{c}
-g S I \\
g S I \\
0 \\
0
\end{array}\right]
\end{gathered}
$$

$$
\mathrm{B}=\left[\begin{array}{cccc}
-(d+u) & 0 & 0 & b \\
0 & -(\alpha+m+d) & 0 & 0 \\
u & \alpha & -d & 0 \\
0 & -m & 0 & (b-d)
\end{array}\right] \text { Now, }
$$

$$
F\left(\phi_{1}\right)-F\left(\phi_{2}\right)=\left[\begin{array}{c}
-g S_{1} I_{1} \\
g S_{1} I_{1} \\
0 \\
0
\end{array}\right]-\left[\begin{array}{c}
-g S_{2} I_{2} \\
g S_{2} I_{2} \\
0 \\
0
\end{array}\right]
$$

Equation (4) is a non-linear system with a bounded coefficient. We set $D(\phi)=\phi_{t}=B \phi+F(\phi)$

To obtain the existence of an optimal control and optimality system the boundedness of solution of the system for finite time is needed and we consider for $u \in U$, there exists a bounded solution.
Now,

$$
\begin{aligned}
\left|F\left(\phi_{1}\right)-F\left(\phi_{2}\right)\right| & =\left|-g S_{1} I_{1}+g S_{2} I_{2}\right| \\
& +\left|g S_{1} I_{1}-g S_{2} I_{2}\right| \\
& =g\left|S_{1} I_{1}-S_{2} I_{2}\right|+g\left|S_{1} I_{1}-S_{2} I_{2}\right| \\
& =2 g\left|S_{1} I_{1}-S_{2} I_{2}\right| \\
& =2 g\left|S_{1} I_{1}-S_{2} I_{1}+S_{2} I_{1}-S_{2} I_{2}\right| \\
& =2 g\left|I_{1}\left(S_{1}-S_{2}\right)+S_{2}\left(I_{1}-I_{2}\right)\right| \\
& \leq 2 g\left(\left|I_{1}\right|\left|S_{1}-S_{2}\right|\right. \\
& \left.+\left|S_{2}\right|\left|I_{1}-I_{2}\right|\right) .
\end{aligned}
$$

We can write,

$$
\begin{gathered}
\left|F\left(\phi_{1}\right)-F\left(\phi_{2}\right)\right| \leq M\left[\left|S_{1}-S_{2}\right|+\left|I_{1}-I_{2}\right|\right] \\
\leq M\left|\phi_{1}-\phi_{2}\right|
\end{gathered}
$$

where, $M=2 g V_{1}$

Also, we get

$$
\begin{aligned}
\left|D\left(\phi_{1}\right)-D\left(\phi_{2}\right)\right| \leq & \|B\|\left|\phi_{1}-\phi_{2}\right|+M\left|\phi_{1}-\phi_{2}\right| \\
& \leq V\left|\phi_{1}-\phi_{2}\right|
\end{aligned}
$$

where, $V=\max (M,\|B\|)<\infty$.

Thus, it follows that the function $D$ is uniformly Lipschitz continuous. From the definition of the control $u(t)$ and the restriction on $S, I, R$ and $N \geq 0$, we see that a solution of the system (4) exists.

\section{B. Existence of the objective functional}

Consider the following optimal control problem

$$
J(u)=\int_{0}^{T} A I(t)+u^{2}(t) d t
$$

Our aim is to minimize the number of infectious individuals and also to minimize the vaccination cost. Infact, the infected people need medical treatment. Here, T indicates the treatment period or vaccination period for which time the infected people keeps under treatment. The value $\mathrm{A}$ is a balancing parameter that establishes the importance and interaction between two factors.

To prove the existence of the objective functional we use the following theorem from Fleming and Rishel [9]. 
Theorem 1: Let

$$
\bar{x}(t)=\left[\begin{array}{c}
x_{1}(t) \\
\vdots \\
x_{n}(t)
\end{array}\right]
$$

be a system of $\mathrm{n}$ state variables, and let $u(t)$ be a control variable with set of admissible controls $U$, that satisfy the following differential equation

$$
x_{i}^{\prime}(t)=g_{i}\left(t, x_{i}(t), u(t)\right) \text { for } i=1, \ldots n
$$

with associated objective functional

$$
J(u)=\int f(t, \bar{x}(t), u(t)) d t
$$

There exists an optimal control which minimizes $J(u)$ if the following conditions are satisfied:

(i) $\mathcal{F}$ is non-empty.

(ii) The control set $U$ must be closed and convex.

(iii) The right hand side of the state system is continuous, is bounded above by a linear combination of the control and the state, and can be written as a linear function of $u$ with coefficients defined by the time and the state.

(iv) The integrand of the objective functional is convex on $U$ and is bounded below by $-C_{2}+C_{1}(u)^{\eta}$, with $C_{1}>0$ and $\eta>1$

We define $\mathcal{F}$ as a class of $\left(S_{0}, I_{0}, R_{0}, N_{0}, u\right)$ such that $u$ is a piecewise function on $[0, T]$ with values in $U$. To prove that $\mathcal{F}$ is nonempty, we will use a simplified version of an existence result in Boyce and DiPrima [10] which is stated below.

Theorem 2: Let each of the functions $F_{1}, \ldots, F_{n}$ and the partial derivatives $\frac{\partial F_{1}}{\partial x_{1}}, \ldots, \frac{\partial F_{1}}{\partial x_{n}}$,

$\ldots, \frac{\partial F_{n}}{\partial x_{1}}, \ldots, \frac{\partial F_{n}}{\partial x_{n}}$ be continuous in a region $\mathcal{R}$ of $t, x_{1}, x_{2}, \ldots, x_{n}$ space defined by $\alpha<t<\beta, \alpha_{1}<x_{1}<$ $\beta_{1}, \ldots, \alpha_{n}<x_{1}<\beta_{n}$, and let the point $\left(t_{0}, x_{1}^{0}, x_{2}^{0}, \ldots, x_{n}^{0}\right)$ be in $\mathcal{R}$. Then there is an interval $\left[t-t_{0}\right]<h$ in which there exists a unique solution $x_{1}=\phi_{1}(t), \ldots, x_{n}=\phi_{n}(t)$ of the system of differential equations

$$
\begin{aligned}
x_{1}^{\prime} & =F_{1}\left(t, x_{1}, \ldots, x_{n}\right), \\
x_{2}^{\prime} & =F_{2}\left(t, x_{1}, \ldots, x_{n}\right), \\
\vdots & \\
x_{n}^{\prime} & =F_{n}\left(t, x_{1}, \ldots, x_{n}\right)
\end{aligned}
$$

that also satisfies the initial conditions

$$
x_{1}\left(t_{0}\right)=x_{1}^{0}, x_{2}\left(t_{0}\right)=x_{2}^{0}, \ldots, x_{n}\left(t_{0}\right)=x_{n}^{0} .
$$

Theorem 3: Let $x_{i}=F_{i}\left(t, x_{1}, \ldots, x_{n}\right)$ for $i \in[1, n]$ be a system of $n$ differential equations with initial conditions $x_{i}\left(t_{0}\right)=x_{i}{ }^{0}$ for $i \in[1, n]$. If each of the functions $F_{1}, \ldots, F_{n}$ and the partial derivatives $\frac{\partial F_{1}}{\partial x_{1}}, \ldots \frac{\partial F_{1}}{\partial x_{n}}, \ldots, \frac{\partial F_{n}}{\partial x_{1}}, \ldots, \frac{\partial F_{n}}{\partial x_{n}}$ are continuous in $\mathcal{R}^{n+1}$ space, then there exists a unique solution $x_{1}=\sigma_{1}(t), \ldots, x_{n}=\sigma_{n}(t)$ that satisfies the initial conditions.

With the help of above two theorems (theorem 2, theorem 3) we try to prove the existence of the optimal control. We show that there exists an optimal control $u^{*}$ that minimizes $J(u)$ over the control set $U$.

Proof of (i): Let

$$
\begin{aligned}
\frac{d S}{d t} & =F_{1}(t, S, I, R, N) \\
\frac{d I}{d t} & =F_{2}(t, S, I, R, N) \\
\frac{d R}{d t} & =F_{3}(t, S, I, R, N) \\
\frac{d N}{d t} & =F_{4}(t, S, I, R, N),
\end{aligned}
$$

where $F_{1}, F_{2}, F_{3}$ and $F_{4}$ buildup the right hand side of the syestem $(3)$. Let $u(t)=C$ for some constant $C . F_{1}, F_{2}, F_{3}$ and $F_{4}$ must be linear and they are also continuous everywhere. Moreover, the partial derivatives of $F_{1}, F_{2}, F_{3}$ and $F_{4}$ with respect to all states are constants and they are also continuous everywhere, so by the above theorem 3 , there exists an unique solution $S=\sigma_{1}(t), I=\sigma_{2}(t), R=\sigma_{3}(t), N=\sigma_{4}(t)$ which satisfies the initial conditions. Therefore, the set of controls and corresponding state variables is non-empty. Hence the condition (i) is satisfied.

Proof of (ii): By definition, $U$ is closed. We take any two controls $u_{1}, u_{2} \in U$ and $\epsilon \in[0,1]$ such that

$$
0 \leq \epsilon u_{1}+(1-\epsilon) u_{2} .
$$

We also observe that $\epsilon u_{1} \leq \epsilon$ and $(1-\epsilon) u_{2} \leq(1-\epsilon)$. Then

$$
\epsilon u_{1}+(1-\epsilon) u_{2} \leq \epsilon+(1-\epsilon)=1
$$

Hence, $0 \leq \epsilon u_{1}+(1-\epsilon) u_{2} \leq 1$ for all $u_{1}, u_{2} \in U$ and $\epsilon \in[0,1]$.

So, $U$ is convex and therefore condition (ii) is satisfied.

Proof of (iii): If we consider,

$$
\begin{gathered}
F_{1} \leq b N-u S \\
F_{2} \leq K_{1} I \\
F_{3} \leq \alpha I+u S \\
F_{4} \leq b N
\end{gathered}
$$

then the following system

$$
\begin{aligned}
\frac{d S}{d t} & =F_{1}(t, S, I, R, N) \\
\frac{d I}{d t} & =F_{2}(t, S, I, R, N) \\
\frac{d R}{d t} & =F_{3}(t, S, I, R, N) \\
\frac{d N}{d t} & =F_{4}(t, S, I, R, N)
\end{aligned}
$$


can be rewritten as,

$\bar{F}(t, \bar{X}, u) \leq \bar{m}\left(t,\left[\begin{array}{c}S \\ I \\ R \\ N\end{array}\right]\right) \bar{X}(t)+\bar{n}\left(t,\left[\begin{array}{c}S \\ I \\ R \\ N\end{array}\right]\right) u(t)$

where

$$
\bar{m}\left(t,\left[\begin{array}{c}
S \\
I \\
R \\
N
\end{array}\right]\right)=\left[\begin{array}{cccc}
0 & 0 & 0 & b \\
0 & K_{1} & 0 & 0 \\
0 & \alpha & 0 & 0 \\
0 & 0 & 0 & b
\end{array}\right]
$$

and

$$
\bar{n}\left(t,\left[\begin{array}{c}
S \\
I \\
R \\
N
\end{array}\right]\right)=\left[\begin{array}{c}
-S \\
0 \\
S \\
0
\end{array}\right]
$$

which gives a linear function of the control $u$ defined by time and state variables. Then we can find out the bound of the right hand side. It is noted that all parameters are constant and greater than or equal to zero. Therefore we can write,

$$
\begin{gathered}
|\bar{F}(t, \bar{X}, u)| \leq|| \bar{m}|||\bar{X}|+|\bar{S}||u(t)| \\
\leq p(|\bar{X}|+|u(t)|)
\end{gathered}
$$

Since $\bar{S}$ is bounded and $\mathrm{p}$ includs the upper bound of the constant matrix. Hence we see that the right hand side is bounded by a sum of the state and the control. Therefore, condition (iii) is satisfied.

\section{Proof of (iv):}

Let us consider that the integrand of the objective functional be $f(u)=A I(t)+u^{2}$. We take two controls $u_{1}, u_{2} \in U$ and $0<\theta<1$. Then we can write,

$u_{1}^{2}-2 u_{1} u_{2}+u_{2}^{2}=\left(u_{1}-u_{2}\right)^{2} \geq 0$

$$
\begin{gathered}
\Longrightarrow u_{1}{ }^{2}+u_{2}{ }^{2} \geq 2 u_{1} u_{2} \\
\Longrightarrow \theta(1-\theta) u_{1}{ }^{2}+\theta(1-\theta) u_{2}{ }^{2} \geq \theta(1-\theta) 2 u_{1} u_{2} \\
\Longrightarrow\left(\theta-\theta^{2}\right) u_{1}{ }^{2}+\left[(1-\theta)-(1-\theta)^{2}\right] u_{2}{ }^{2} \geq 2 \theta(1-\theta) u_{1} u_{2} \\
\Longrightarrow \theta u_{1}^{2}+(1-\theta) u_{2}{ }^{2} \geq \theta^{2} u_{1}^{2}+(1-\theta)^{2} u_{2}{ }^{2}+2 \theta(1- \\
\theta) u_{1} u_{2} \\
\Longrightarrow \theta u_{1}{ }^{2}+(1-\theta) u_{2}{ }^{2} \geq\left[\theta u_{1}+(1-\theta) u_{2}\right]^{2} \\
\Longrightarrow A I(t)+\theta u_{1}{ }^{2}+(1-\theta) u_{2}{ }^{2} \geq A I(t)+\left[\theta u_{1}+(1-\theta) u_{2}\right]^{2} \\
\Longrightarrow \quad A I(t)[\theta+(1-\theta)]+\theta u_{1}{ }^{2}+(1-\theta) u_{2}{ }^{2} \geq \\
A I(t)+\left[\theta u_{1}+(1-\theta) u_{2}\right]^{2} \\
\Longrightarrow \theta A I(t)+A I(t)(1-\theta)+\theta u_{1}{ }^{2}+(1-\theta) u_{2}{ }^{2} \geq \\
A I(t)+\left[\theta u_{1}+(1-\theta) u_{2}\right]^{2}
\end{gathered}
$$$$
\Longrightarrow \theta f\left(u_{1}\right)+(1-\theta) f\left(u_{2}\right) \geq f\left(\theta u_{1}+(1-\theta) u_{2}\right)
$$

which implies that $f(u)$ is convex on $U$

Now we will show that

$\left(P(u) \geq-C_{2}+C_{1}(u)^{\eta}\right.$, with $\eta>1, C_{1} \geq 0$

Here,

$$
\begin{gathered}
J(u)=A I(t)+u^{2} \\
J(u) \geq-A I(t)+u^{2} \\
=-C_{2}+C_{1} u^{2}
\end{gathered}
$$

where $C_{2}>0$ which depends on upper bounds of $I(t)$. We can also see that $\eta=2>1, C_{1}>0$. Therefore, condition (iv) is also satisfied. From the above discussion the existence of the objective functional has been established.

\section{Characterization of the Optimal Control}

By applying Pontryagin's Maximum Principle [11] Hamiltonian $(H)$, we will derive the necessary conditions for the optimal control. While considering the optimal system, we will ignore the recovered class as the variable $\mathrm{R}$ appears only in the recovered class. Therefore our derived Hamiltonian $(H)$ can be defined as follows

$$
\begin{aligned}
& H(S, I, t)=A I+u^{2}+\lambda_{s}(b N-g S I-d S-u S) \\
& +\lambda_{I}(g S I-(\alpha+d+m) I)+\lambda_{N}((b-d) N-m I)
\end{aligned}
$$

Where, $\lambda_{S}, \lambda_{I}, \lambda_{N}$ are the associated adjoints for the state $S, I, N$ respectively. Then we differentiate the Hamiltonian with respect to the state variable $S, I, N$ respectively and we consider $\lambda_{S}(T)=\lambda_{I}(T)=\lambda_{N}(T)=0$ because at final stage they do not have fixed value.

$$
\begin{aligned}
\lambda_{S}^{\prime} & =\lambda_{S}(d+g I+u)-\lambda_{I} g I \\
\lambda_{I}^{\prime} & =\lambda_{S} g S+\lambda_{I}(\alpha+d+m)-\lambda_{I} g S-A \\
\lambda_{N}^{\prime} & =\lambda_{S} b+\lambda_{N}(b-d)
\end{aligned}
$$

For the optimal condition we differentiate the Hamiltonian with respect to $u$ at $u=u^{*}$ we get,

$$
\begin{aligned}
\left.\frac{\partial H}{\partial u}\right|_{u=u^{*}} & =0 \\
\Longrightarrow u^{*} & =\frac{S \lambda_{S}}{2}
\end{aligned}
$$

where $u^{*}$ denotes the optimal control. By standard control arguments involving the bounds on the control, we get

$$
u^{*}=\left\{\begin{array}{lr}
\frac{S \lambda_{S}}{2} & \text { if } 0 \leq \frac{S \lambda_{S}}{2} \leq 0.9 \\
0 & \text { if } \frac{S \lambda_{S}}{2}<0 \\
0.9 & \text { if } \frac{S \lambda_{S}}{2}>0.9
\end{array}\right.
$$

In compact notion:

$$
u^{*}=\min \left[0.9, \max \left[0, \frac{S \lambda_{S}}{2}\right]\right] .
$$



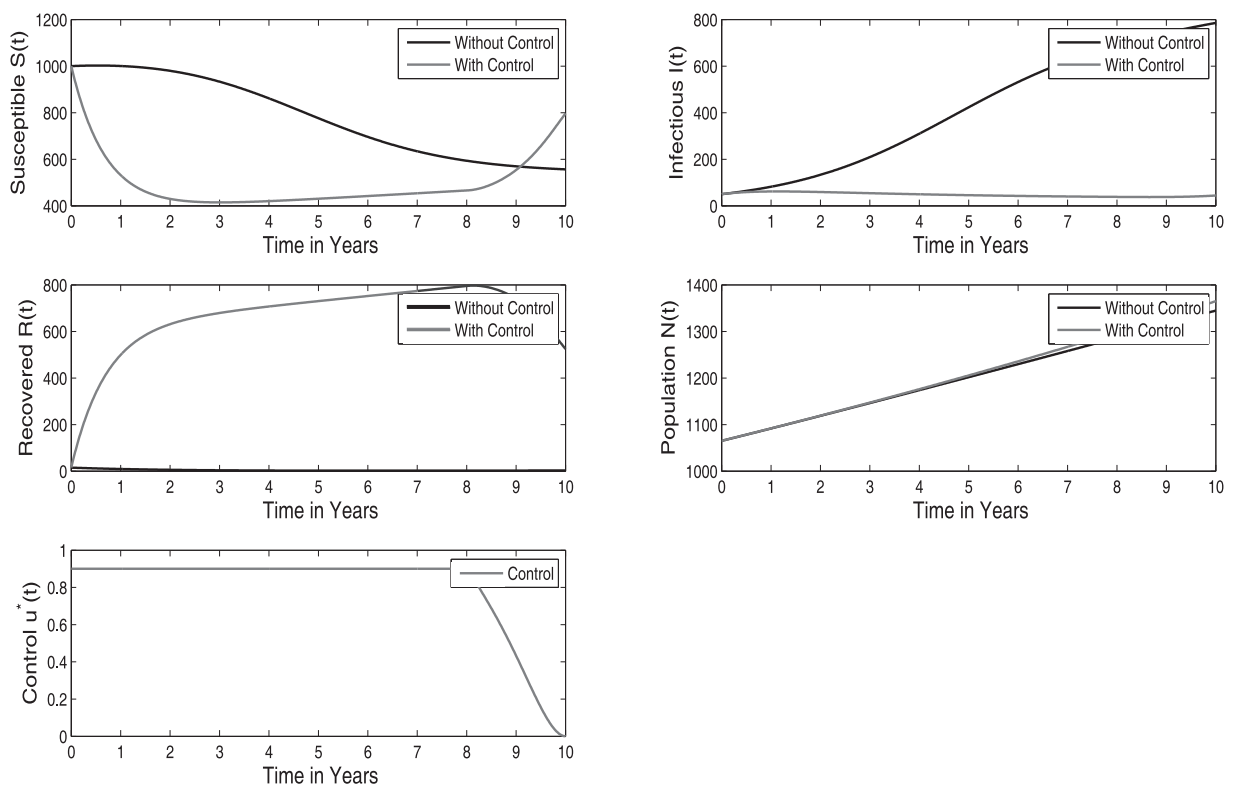

Fig. 1: SIR model with vaccination and without vaccination, parameter values are taken from Table 1.
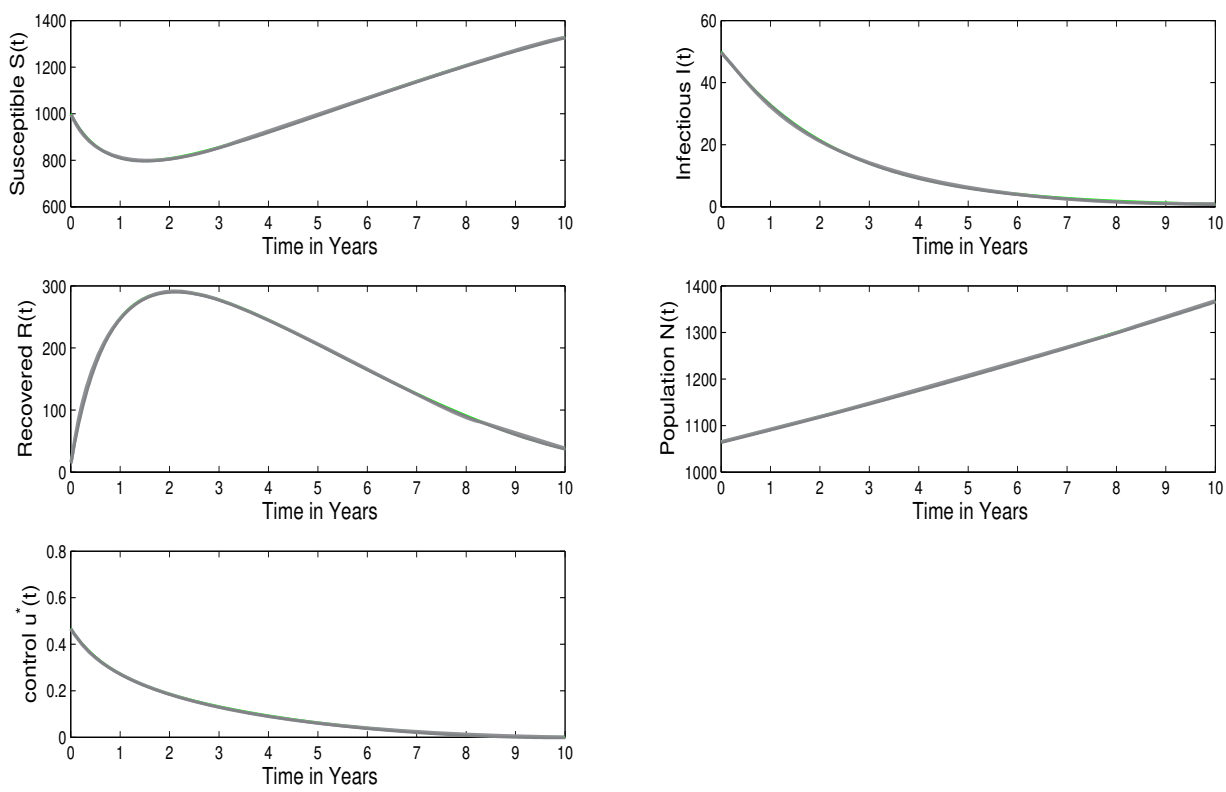

Fig. 2: SIR model with vaccination changing parameter $g=.0001$ and other parameter values are taken from Table1.

\section{Optimality System}

The resulting optimality system is given below:

State equations:

$$
\begin{aligned}
S^{\prime}(t) & =b N(t)-g S(t) I(t)-d s(t)-u(t) S(t) \\
I^{\prime}(t) & =g S(t) I(t)-(\alpha+m+d) I(t) \\
R^{\prime}(t) & =\alpha I(t)-d R(t)+u(t) S(t) \\
N^{\prime}(t) & =(b-d) N(t)-m I(t)
\end{aligned}
$$

with initial conditions,

$$
S(0)=S_{0} \geq 0, I(0)=I_{0} \geq, R(0)=R_{0} \geq 0, N(0)=N_{0}
$$

Adjoint equations:

$$
\begin{aligned}
\lambda_{S}^{\prime} & =\lambda_{S}(d+g I+u)-\lambda_{I} g I \\
\lambda_{I}^{\prime} & =\lambda_{S} g S+\lambda_{I}(\alpha+d+m)-\lambda_{I} g S-A \\
\lambda_{N}^{\prime} & =\lambda_{S} b+\lambda_{N}(b-d)
\end{aligned}
$$



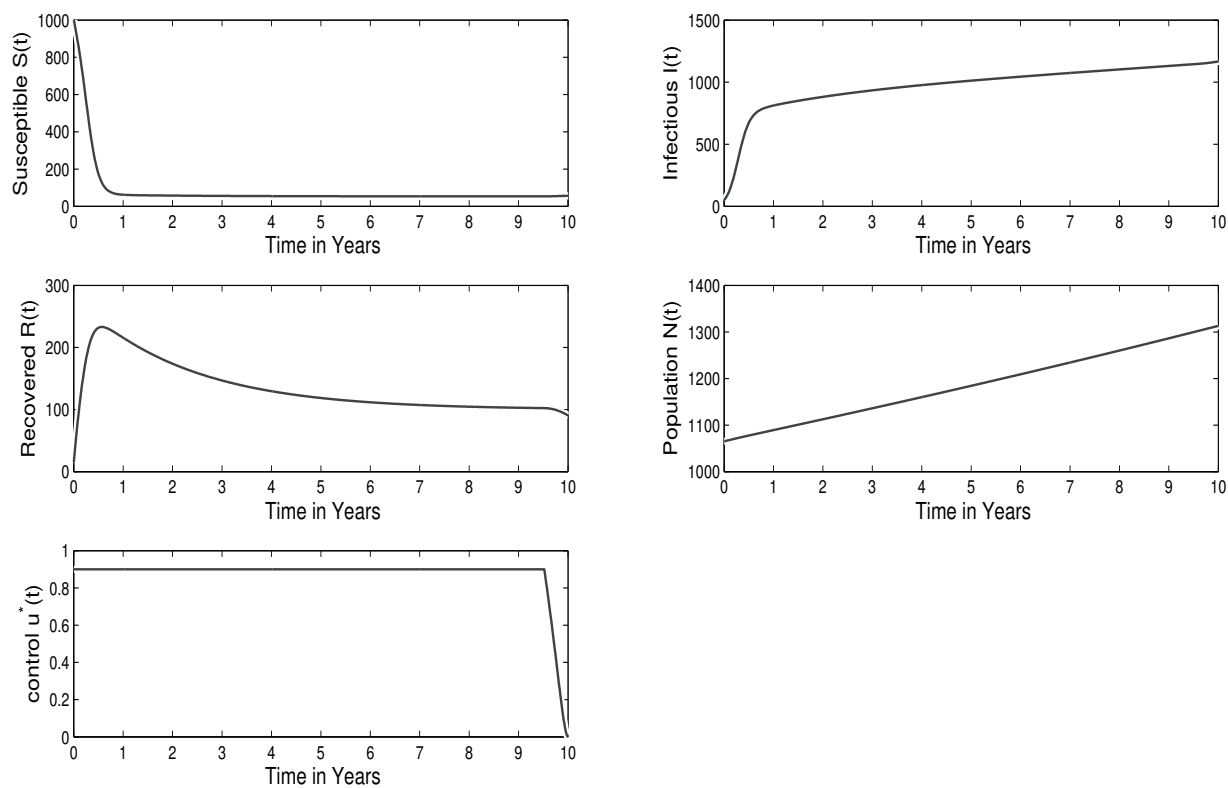

Fig. 3: SIR model with vaccination changing parameter $g=.01$ and other parameter values are taken from Table 1.
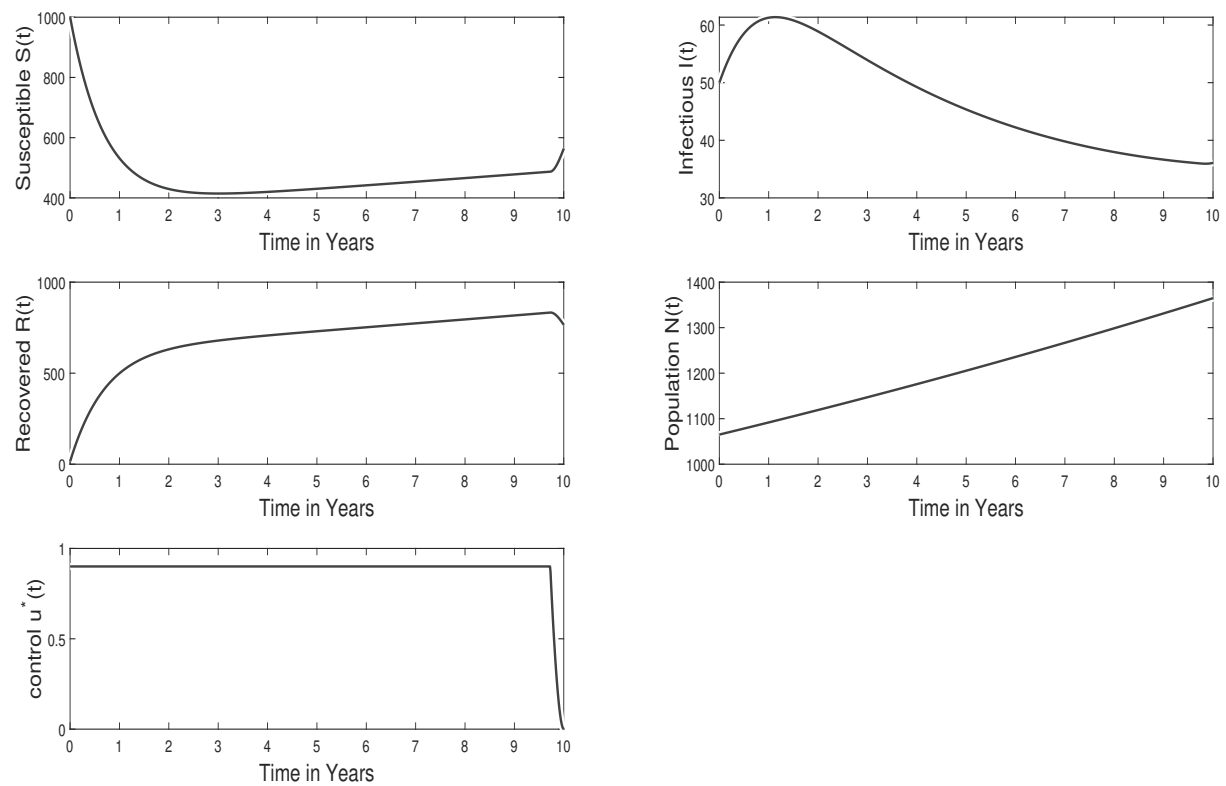

Fig. 4: SIR model with vaccination and changing parameter $\mathrm{A}=3$ and other values are taken from Table 1.

Transversality equations:

$$
\lambda_{S}(T)=\lambda_{I}(T)=\lambda_{N}(T)=0
$$

Characterization of the optimal control $u *$ :

$$
u^{*}=\left\{\begin{array}{lr}
\frac{S \lambda_{S}}{2} & \text { if } 0 \leq \frac{S \lambda_{S}}{2} \leq 0.9 \\
0 & \text { if } \frac{S \lambda_{S}}{2}<0 \\
0.9 & \text { if } \frac{S \lambda_{S}}{2}>0.9
\end{array}\right.
$$

\section{Numerical Result Simulations AND DISCUSSIONS}

Here we solve the state equations with initial conditions numerically using Runge-Kutta method of fourth order forward in timing. After solving the system without vaccination, the system with control characterization is solved numerically to illustrate our control results. This iterative process terminates when current state, adjoint and control values are sufficient close to successive values. 

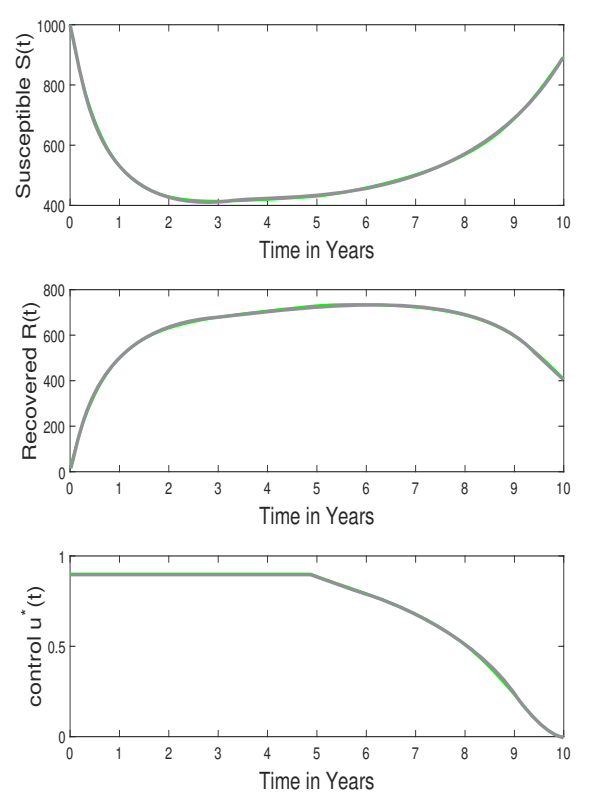
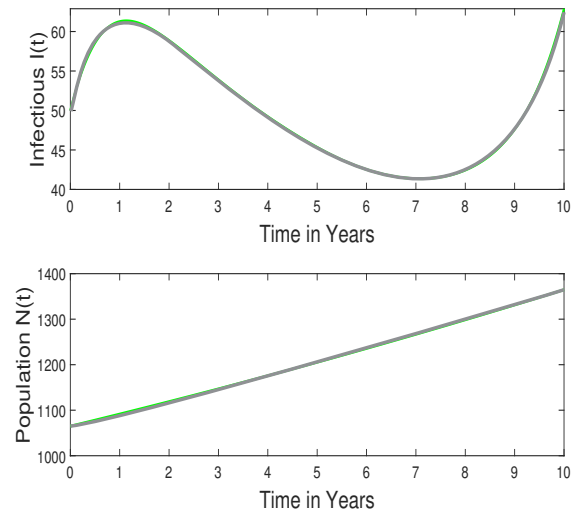

Fig. 5: SIR model with vaccination changing parameter $A=0.03$ and other parameter values are taken from Table 1.

By Using MATLAB, We execute the numerical solutions to the optimality system. We depict the graphs of the state variables with and without control strategy to monitor the effect of control strategy. We monitor the affect of contact rate if the control strategy is applied in delay. we also monitored how the weight parameter affects the control strategy.

The parameter values used in the simulations are shown in Table 1.

\section{TABLE I: DESCRIPTION AND PARAMETER VALUES} OF THE SIR MODEL :

\begin{tabular}{lll}
\hline Variable & Description & Initial Values \\
\hline \hline$S_{0}$ & initial susceptible individuals & $1000[7]$ \\
$I_{0}$ & initial infected individuals & $50[7]$ \\
$R_{0}$ & initial recovered individuals & $15[7]$ \\
& & \\
\hline \hline Parameters & Definition of Parameters & Initial values \\
\hline \hline$b$ & birth rate & $0.525[7]$ \\
$d$ & death rate & $0.5[7]$ \\
$g$ & incidence coefficient & $.001[7]$ \\
$\alpha$ & recovery rate & 0.002 [assumed] \\
$m$ & disease induced death rate & 0.005 [assumed] \\
$A$ & weight parameter & $0.1[7]$ \\
$T$ & number of years & $10[$ assumed] \\
\hline \hline
\end{tabular}

Now we represent the results of our analysis in several steps.

We next solve the optimality system considering the unlimited vaccines, that is, we can vaccinate the susceptible individuals as many as we choose during the 10 years.

Figure 1 shows the importance of vaccination to control the disease.we can see from the graph that control strategy is much more effective to reduce susceptible and infected individual, also to increase recovered individual. It is clear that before vaccination the number of recovered individual was negligible and after applying vaccination it increased remarkably. Overall, there is an increase in the total number of population after vaccination.

Figure 2 and Figure 3 show the effectiveness of control of various disease transmission rate. It is monitored from the figures that for high transmission rate $(g=0.01)$ the control has to work for longer period of time as infected individual increases whereas for low transmission rate $(g=0.0001)$, the recovered individual increases for the effectiveness of the control.This also establishes the importance of early vaccination.

Now we are showing the way of the weight parameter affecting the control strategy.

Figure 4 depicts that when weight parameter is high ( $\mathrm{A}=3$ ), the recovered individuals increase gradually and the susceptible population starts to decrease.

Figure 5 shows that for low weight parameter $(A=0.03)$, the number of infected individuals increases gradually for the first two years.It is also observed that the number of infected individuals started increasing with the decrease of the effectiveness of the control.

\section{CONCLUSION}

Using optimal control technique we have observed that a vaccination program minimizes the number of infected individuals and the overall cost of vaccination during a time interval. After using optimal control technique it is noticed that the population of recovered individuals with control is 
increased within a short period.The behavior of the solution of all cases urge to apply vaccination strategy in early stage of diseases.

\section{REFERENCES}

[1] H.Gaff, E. Schaefer. Optimal control applied to vaccination and treatment strategies for various epidemiological models. Mathematical Biosciences and Engineering, 2009, 6: 469-492.

[2] K. Fister, J. Donnelly. Immunotherapy: An optimal control theory approach. Mathematical Bio sciences and Engineering, 2005, 499-510.

[3] K. Wang, A. Fan, A. Tores. Global properties of an improved hepatitis B virus model. Nonlinear Analysis: Real World Applications, 2009. Doi:10.1016/j.nonrwa.2009.11.008.

[4] G. Zaman, Y. Kang, I. Jung. Stability analysis and optimal vaccination of an sir epidemic model. BioSystems, 2008, 93: 240-249.

[5] D. Kirschner, S. Lenhart, S. SerBin. Optimal control of the chemotherapy of HIV. Journal of Mathematical Biology, 1997, 35: 775-792.

[6] T. Zhang, Z. Teng. Global behavior and permanence of sirs epidemic model with time delay. Nonlinear Analysis: Real World Applications (2008), 9: pp. 1409-1424.

[7] Lenhart S. and J. Workman. Optimal Control Applied to Biological Models, Boca Raton, Chapman Hall/CRC, 2007.

[8] (MR2744727) R. M. Neilan and S. Lenhart. An Introduction to Optimal Control with an Application in Disease Modeling, DIMACS Series in Discrete Mathematics, 75 (2010), pp: 67-81.

[9] Fleming, W. H. and R. W. Rishel. Deterministic and Stochastic Optimal Control, Springer Verlag, New York, 1975.

[10] William E. Boyce and Richard C. DiPrima. Elementary Differential Equations and Boundary Value Problems, John Wiley and Sons, New York, 2009.

[11] Pontryagin, L. S. V. G. Boltyanskii, R. V. Gamkrelize, and E. F. Mishchenko. The Mathematical Theory of Optimal Processes, New York, Wiley, 1962.

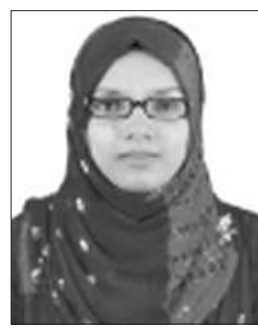

Jakia Sultana was born in Tangail district. She obtained her B.S. ( Hons.) degree in Mathematics and M.S. degree in Applied Mathematics from Department of Mathematics, University of Dhaka in 2013 and 2015 respectively.She worked as a lecturer in Sonargaon University from 4th May, 2015 to 15th January, 2016. she joined Green University of Bangladesh as a lecturer on 16th January, 2016. Currently she is serving as a Senior Lecturer (Mathematics) in the department of Computer Science and Engineering (CSE) in the Green University of Bangladesh (GUB). Her research interests include mathematical modeling in Biology, mathematical fluid dynamics, Optimal control technique, dynamical systems, Operations Research and Optimization.

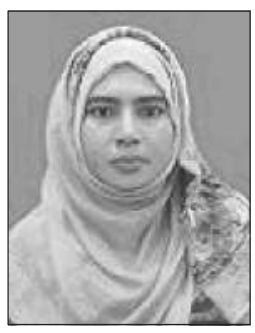

Samiha Islam Tanni was Born in Manikganj district in 1989. She received her BS degree in Mathematics and MS degree in Applied Mathematics in 2013 and 2015 respectively from University of Dhaka (DU). Currently she is serving as a lecturer (Mathematics) in the department of Computer Science and Engineering (CSE) in the Green University of Bangladesh (GUB) since September, 2018. She was also a field researcher of a project named Simon project which was organised by the primak group. Her research interests include Mathematical Modeling in Biology, Optimal Control Technique for reducing infectious diseases, Dynamical System etc.

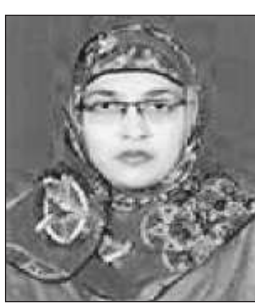

fluid dynamics.
Shamima Islam was born in Chandpur district in 1987. She received her BS degree in Mathematics and MS degree in Applied Mathematic in 2011 and 2013 respectively from University of Dhaka (DU). Currently she is serving as a Senior Lecturer (Mathematics) in the department of Computer Science and Engineering (CSE) in the Green University of Bangladesh (GUB) since 05 September, 2014. Her research interests include mathematical modeling and simulation of fluidic phenomena, mathematical 